\title{
Evaluation of a novel atrial retractor for exposure of the mitral valve in a porcine model
}

\author{
Eric Bean, MS, ${ }^{\mathrm{a}}$ Guillaume Chanoit, DVM, MS, ${ }^{\mathrm{b}}$ Shaphan Jernigan, MS, ${ }^{\mathrm{a}}$ Gil Bolotin, MD, PhD, ${ }^{\mathrm{c}} \mathrm{Jason}$ Osborne, PhD, ${ }^{\mathrm{d}}$ and \\ Gregory Buckner, $\mathrm{PhD}^{\mathrm{a}}$
}

Objectives: To describe a novel atrial retractor and compare 2 methods of intraoperative left atrial retraction for
minimally invasive mitral valve repair.

\begin{abstract}
Methods: Left atrial retraction was performed on 5 swine cadavers to evaluate performance (percent of mitral valve annulus accessible), complications encountered, ease of use, and surgical time for the minimally invasive atrial retractor and a HeartPort atrial retractor.

Results: Estimated accessibilities were 93.0\% (standard error $=3.2$ ) and 92.7\% (standard error $=3.3$ ) for the HeartPort and minimally invasive atrial retractor retractors, respectively, with a difference of $0.3 \%$ (standard error $=2.2 \%, P=.8832, d f=34$ ). Tissue damage occurred in 1 case for the minimally invasive atrial retractor and 2 cases for the HeartPort retractor. The mean surgical times for retractor placement and mitral valve annulus exposure were 107.4 and 39.2 seconds for the HeartPort and minimally invasive atrial retractor retractors, respectively, with a difference of 68.2 seconds $(P=.0092, d f=4)$.

Conclusions: The minimally invasive atrial retractor is a suitable alternative for atrial retraction compared with standard techniques of retraction. It provides comparable exposure of the mitral valve annulus, is less time consuming to place, provides subjectively more working volume within the left atrium, and has the advantage of minimal atriotomy incision length and customizable retraction.
\end{abstract}

As minimally invasive cardiac surgery continues to improve ${ }^{1,2}$ there is an increased need for tissue retractors that can be deployed and maneuvered through smaller incisions. Tissue retraction is critical for proper exposure of operative fields limited by these incisions. Furthermore, there exists a need to overcome limitations of current minimally invasive cardiac procedures, which include increased cardiopulmonary bypass time and increased surgical skill requirements. ${ }^{2}$ As Kypson noted, truly endoscopic cardiac procedures require the development of specialized retractors. ${ }^{1}$

Surgical retraction has been studied in recent years within the realm of cardiac surgery and other specialties. Smith and colleagues recently evaluated the novel EndoWrist atrial retractor (Intuitive Surgical Inc, Sunnyvale, Calif) in an 18-patient study of mitral valve repairs. ${ }^{3}$ Coupled to the da Vinci surgical robot, the EndoWrist retractor can be inserted through a small port, then expanded and maneuvered within the chest cavity. In an interventional case series, Akman and

From the Department of Mechanical and Aerospace Engineering ${ }^{\mathrm{a}}$ and College of Veterinary Medicine, ${ }^{\mathrm{b}}$ North Carolina State University, Raleigh, NC, the Academic Hospital Maastricht, ${ }^{\mathrm{c}}$ Maastricht, the Netherlands, and Department of Statistics, ${ }^{\mathrm{d}}$ North Carolina State University, Raleigh, NC.

This work was funded by a grant from the National Institutes of Health (NIH), National Heart, Lung, and Blood Institute (grant number R01 HL075489-01A1).

Received for publication June 21, 2008; revisions received Aug 8, 2008; accepted for publication Aug 18, 2008.

Address for reprints: Guillaume Chanoit, DVM, MS, College of Veterinary Medicine, North Carolina State University, 4700 Hillsborough St, Raleigh, NC 27606 (E-mail: guillaume_chanoit@ncsu.edu).

J Thorac Cardiovasc Surg 2008;136:1492-5

$0022-5223 / \$ 34.00$

Copyright (C) 2008 by The American Association for Thoracic Surgery doi:10.1016/j.jtcvs.2008.08.030 colleagues compared 4 methods of intraoperative pupil dilation in eyes by measuring performance (pupil size achieved), complications, and added surgical time. ${ }^{4}$

In the case of mitral valve procedures, atrial retraction is commonly performed using a 2-piece blade retractor, such as the HeartPort retractor (Cardiovations, Ethicon Inc, Somerville, NJ), that must be assembled intracorporeally. ${ }^{5,6}$ The blade dimensions (up to $45 \mathrm{~mm}$ by $70 \mathrm{~mm}$ ) necessitate larger atrial incisions; furthermore, the 2-piece design complicates deployment. ${ }^{3}$ Research at North Carolina State University's Electro-Mechanics Research Lab has led to the development of a minimally invasive atrial retractor (MIAR). ${ }^{7}$ Research goals have been to design a retractor that can be deployed through a single small atrial incision $(<2 \mathrm{~cm})$ yet enhance visualization and surgical access to the mitral valve as compared with conventional retractors.

To accomplish these aims, using a protocol similar to Akman and colleagues, ${ }^{4}$ the present study evaluated the effectiveness, placement time, ease of use, and complications for the MIAR compared with the conventional retractor and method (HeartPort) for use during minimally invasive mitral valve surgery.

\section{MATERIALS AND METHODS}

A systematic design process involving computer-aided design modeling, rapid prototyping, and surgical evaluation using explanted porcine hearts and animal cadavers facilitated the development of a highly functional MIAR prototype. The MIAR combines desirable attributes of conventional blade retractors with extendable nitinol appendages that conform to variations in atrial anatomy. The prototype features a narrow $(12 \times 60 \mathrm{~mm})$ but otherwise conventional retractor blade with 2 key features: (1) nitinol appendage deployment and (2) blade pitch adjustment (Figure 1). The 


\section{Abbreviations and Acronyms}

MIAR $=$ minimally invasive atrial retractor

$\mathrm{MV}=$ mitral valve

MVA $=$ mitral valve annulus

operator can actively adjust blade pitch after insertion between 0 degrees (blade inline with instrument axis) and 90 degrees (blade normal to instrument axis). Each of the 4 appendages can be individually deployed. The superelastic properties of the nitinol wire allow it to be deployed and retracted within the blade with no permanent deformation.

\section{Testing Protocol}

To comprehensively test the effectiveness of the MIAR, left atrial retraction procedures were performed on 5 swine cadavers obtained from a local slaughterhouse. Surgeries were performed at the College of Veterinary Medicine at North Carolina State University, by one of the investigators (G.C.). Each surgery consisted of the working incisions and approach required for a standard minimally invasive surgery (MIS) mitral valve (MV) repair: a right anterolateral thoracotomy at the 4th intercostal space and a left atriotomy.

Upon completion of the working incisions, the MV annulus (MVA) was exposed with either retractor (MIAR and a modified $25 \times 40-\mathrm{mm}$ HeartPort). The retractor order for each animal trial was randomized. Atriotomy length was sized to accommodate the width of the HeartPort retractor. To accommodate the small size of porcine atria, a standard HeartPort retractor $(35 \times 40-\mathrm{mm})$ blade was professionally milled to a width of $25 \mathrm{~mm}$ (dimensions after milling: $25 \pm 0.2 \times 40 \mathrm{~mm}$ ) for use on each pig during the trials.

Retractor placement time was defined as the time interval between the completion of the atriotomy and the completion of retractor stabilization with acceptable exposure of the MVA and subvalvular apparatus. Endoscopic images of the MVA were recorded for postsurgical image processing (Figure 2).

\section{Data Analysis}

MV exposure was calculated via a custom MATLAB (The MathWorks, Natick, Mass) image processing script (Figure 2, $B$ and $C$ ). One image from each retraction procedure (1 image per retractor, 2 per pig) featuring exposure of the MVA was distributed to 4 cardiac surgeons and 1 investigator (G.C.). Using a graphical user interface, each surgeon indicated a minimum of 5 best-fit points along the perimeter of the MVA (Figure 2, B). Additionally, the surgeons marked anatomic features that block MVA visualization and/or access. The edges of occluding retractor geometries were indicated by the authors.
For each postprocessed image, the MATLAB script calculated the exposed percent of the MVA. The M-script fits a least-squares ellipse to the points a surgeon has designated at the MVA then plots the ellipse on the surgical image for verification ${ }^{8}$ (Figure 2,B). A similar algorithm fits continuous lines to occluding object boundaries (anatomic features as indicated by the surgeons and retractor geometries) and plots the lines on the surgical image (Figure 2, B). Areas between occluding structures and the MVA ellipse were considered as occluded (Figure 2, $C$ ). Percent accessibility was calculated by dividing accessible area by the total MVA area.

MV exposure was compared by calculating the mean difference in percent accessibility between the MIAR and HeartPort methods for each surgery and the mean and standard deviation for all surgeries.

\section{Statistical Analysis}

The additive mixed model was chosen to test the hypothesis that the long-run mean MVA accessibility is the same for the prototype retractor as for the conventional retractor. Additionally, separate paired $t$ tests were conducted for each pig, using the pairs of measurements from the 5 surgeons. A significance level of .05 was used for all hypothesis tests regarding the effects of retractor type, with $P$ values given for completeness. To test the hypothesis that the mean placement time was equal for the 2 retractor types, using the paired placement times observed on a sample of 5 pigs, a paired $t$ test was conducted.

\section{RESULTS}

Five minimally invasive atriotomies were successfully completed on nonsurvival porcine models with minimal complications using the standard minimally invasive MV repair approach via the 4th right intercostal space. Fitting the additive mixed model to these data led to estimated accessibilities of $93.0 \%$ (standard error $=3.2$ ) and $92.7 \%$ ( standard error $=3.3$ ) for HeartPort and MIAR, respectively, with a difference of $0.3 \%$ (standard error $=2.2 \%, P=$ $.8832, d f=34)$. Tissue damage occurred in 1 case for the MIAR and 2 cases for the HeartPort retractor.

The mean placement times for the HeartPort and MIAR retractors were 107.4 seconds and 39.2 seconds, respectively, for a difference of 68.2 seconds (standard error $=$ $14.4, P=.0092, d f=4$ ). Both retraction methods were effective procedures for the mechanical retraction of the left atrium during MV repair. The MIAR retractor method is the least time-consuming technique of customizable retraction. Quantitative results are summarized in Table 1,
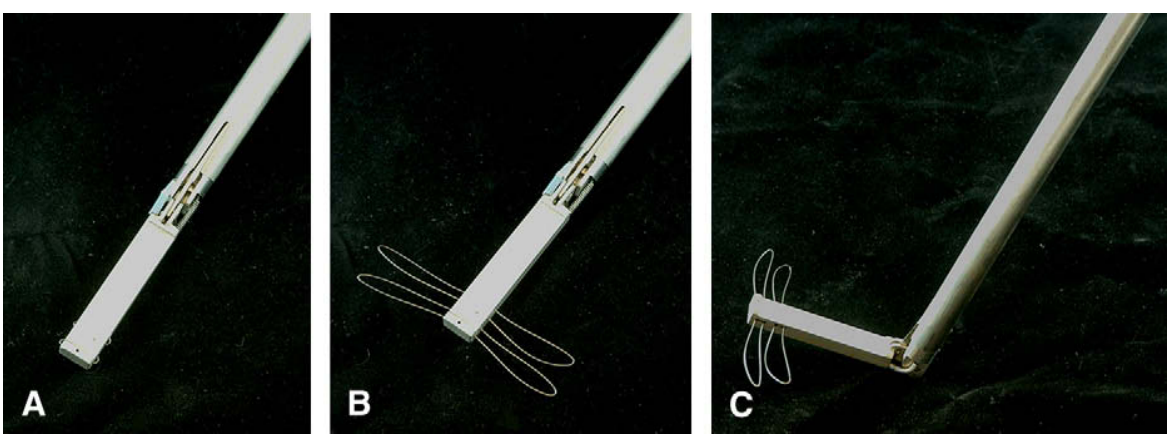

FIGURE 1. Demonstration of minimally invasive atrial retractor nitinol appendage deployment and blade pitch features. A, Appendages undeployed. B, Appendages deployed. C, Blade pitched to approximately 90 degrees. 

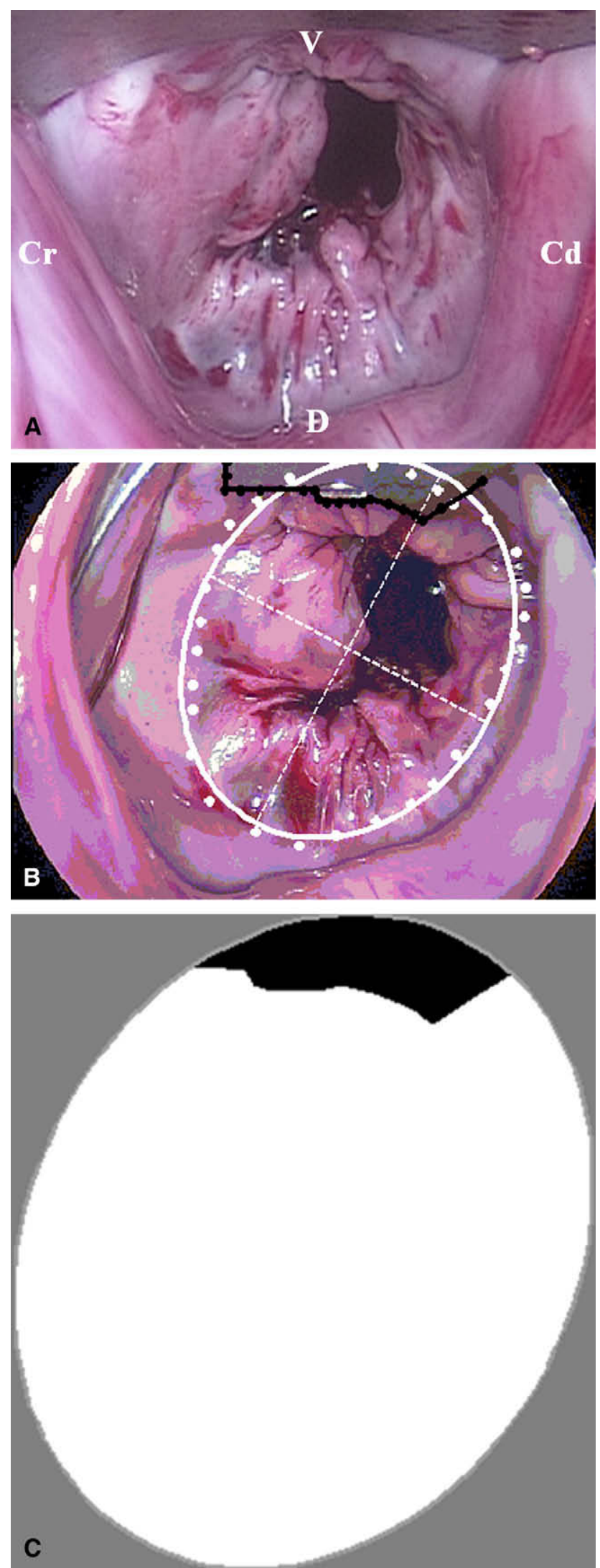

where differences are expressed relative to the HeartPortretractor.

Localized damage, likely caused by high pressure on tissue and device repositioning, consisted of longitudinal tears in the endocardium surrounding the annulus and was noted on 2 pigs: 1 instance for the MIAR and 2 for the HeartPort retractor.

\section{DISCUSSION}

The results from the MIAR animal trials are promising for the near-term development of a totally endoscopic atrial retractor and for a variable-geometry minimally invasive retractor that can be applied to a broad spectrum of surgical cases. Although decreases in retractor placement time during the animal trials are small ( $\sim 1$ minute), reduction in overall MV procedure times could be much greater in a clinical trial. For example, Smith and colleagues reported an approximate 10-minute reduction in crossclamp and cardiopulmonary bypass times in their EndoWrist retractor study. ${ }^{3}$ Slight variations in cardiopulmonary bypass times may not be important for young or otherwise healthy patients but can be critical for elderly or compromised patients. In some cases, the risks of increased cardiopulmonary bypass times do not justify the benefits of minimally invasive surgery. ${ }^{2}$

In this study, the MIAR was introduced through the thoracotomy; however, its design allows for insertion through a 13-mm trocar and thus allows its usage in a totally endoscopic procedure. A future design might benefit from a smaller profile and thus introduction through a smaller incision.

Although both retractors sufficiently retracted the atrial wall at the retractor blade, the MIAR did, in some cases, provide better retraction at the right and left of the endoscopic view. For pigs 2 and 4, the MIAR retractor eliminated an anatomic fold obstructing the mitral annulus (Figure 3). This further retraction would provide not only greater visualization and access to the mitral valve but also a larger working volume for the surgical tools within the left atrium.

In general, the nitinol appendages performed as desired; however, their functionality might be increased by decreasing wire stiffness or designing precurved nitinol appendages. These modifications would allow them to conform more readily to the curvature of the left atrial wall.

Results, namely accessibility data, could be affected by the depth of retractor placement into the left atrium. The MIAR design allowed it to be placed considerably closer to the MVA in pigs 2 and 5 than the HeartPort retractor.

FIGURE 2. Image analysis. A, Retraction with the HeartPort retractor. B, Structure identification and curve-fitting (retraction with the minimally invasive atrial retractor): mitral valve annulus shown in white, retractor points shown in black. C, isolation of occluded (black) and accessible (white) left atrial area for image processing. $V$, Ventral; $C r$, cranial; $C d$, caudal; $D$, dorsal. 
TABLE 1. Quantitative results of animal trials

\begin{tabular}{|c|c|c|c|c|c|c|c|c|c|}
\hline \multirow[b]{2}{*}{ Pig } & \multicolumn{3}{|c|}{ MVA exposure (\%) } & \multicolumn{3}{|c|}{ Placement time (s) } & \multicolumn{3}{|c|}{ Tissue damage } \\
\hline & HP & MIAR & Diff & HP & MIAR & Diff & HP & MIAR & Diff \\
\hline 1 & $97.9 \pm 2.2$ & $96.0 \pm 1.7$ & $-1.9 ; \mathrm{P}=.378$ & $74 *$ & 37 & -37 & $0^{*}$ & 0 & 0 \\
\hline 2 & $85.2 \pm 18.8$ & $83.8 \pm 2.1$ & $-1.4 ; P=.908$ & $153 *$ & 57 & -96 & $0 *$ & 0 & 0 \\
\hline 3 & $98.6 \pm 2.9$ & $98.2 \pm 2.9$ & $-0.4 ; \mathrm{P}=.585$ & 92 & $37 *$ & -55 & 1 & $1 *$ & 0 \\
\hline 4 & $88.0 \pm 8.3$ & $95.4 \pm 7.3$ & $+7.4 ; \mathrm{P}=.024$ & 141 & $32 *$ & -109 & 1 & $0^{*}$ & -1 \\
\hline 5 & $96.0 \pm 7.6$ & $90.7 \pm 7.2$ & $-5.3 ; \mathrm{P}=.389$ & $77 *$ & 33 & -34 & $0 *$ & 0 & 0 \\
\hline Mean \pm SD & $93.0 \pm 6.1$ & $92.7 \pm 6.0$ & $-0.3 ; \mathrm{P}=.883$ & $107.4 \pm 37.0$ & $39.2 \pm 10.2$ & $-68.2 ; P=.009$ & $0.4 \pm 0.5$ & $0.2 \pm 0.4$ & $-0.2 ; P=.374$ \\
\hline
\end{tabular}

$M V A$, mitral valve annulus; $H P$, HeartPort; $M I A R$, minimally invasive atrial retractor; $D i f f$, difference; $S D$, standard deviation. *Indicates the first retractor to be placed in each pig.

Although this deeper positioning can cause some obstruction of the MVA (and thus negatively affect the exposure results of this study), it can also provide benefits, such as elimination of anatomic folds and enlargement of the accessible left atrial volume.
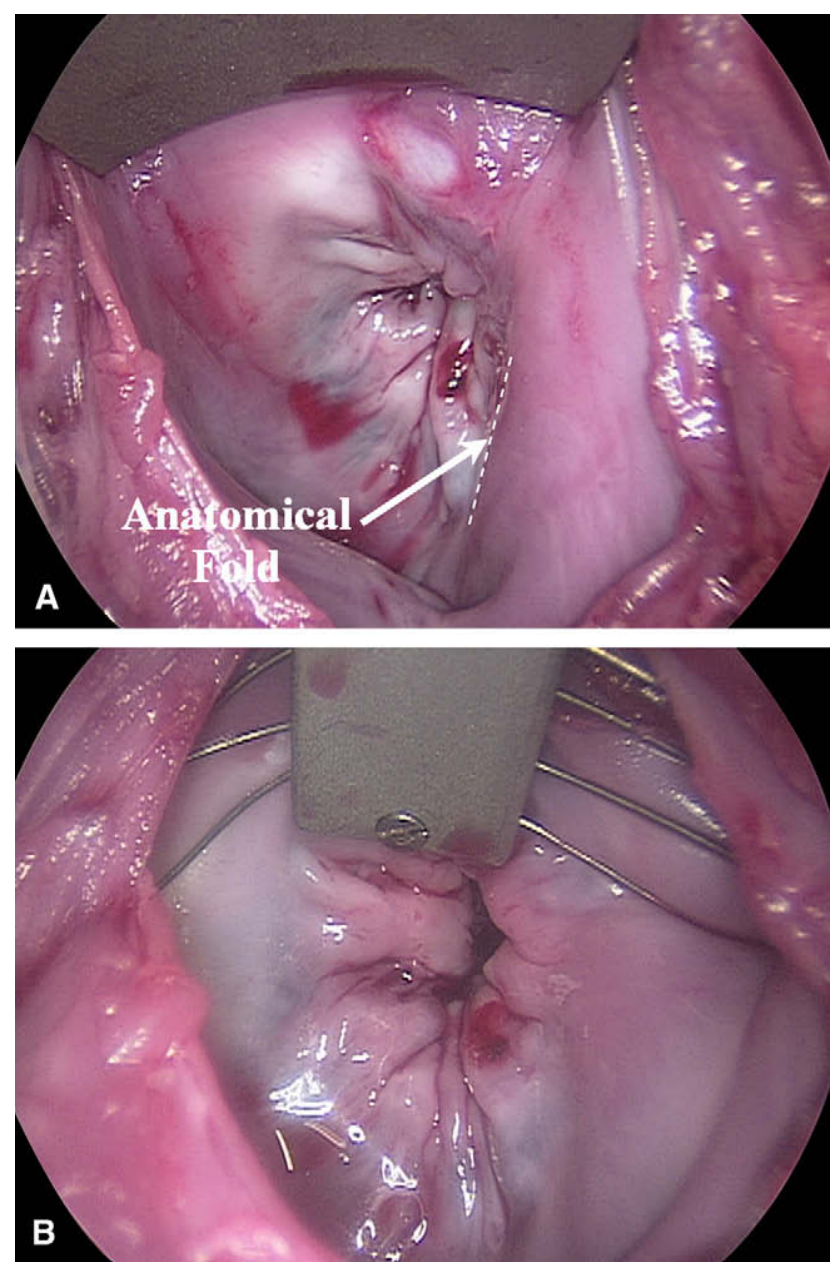

FIGURE 3. Elimination of an anatomic fold with the minimally invasive atrial retractor on pig 2. A, Presence of fold with the HeartPort retractor. $\mathrm{B}$, Absence of fold with the minimally invasive atrial retractor.

\section{CONCLUSION}

This study has demonstrated that the MIAR retractor is a suitable alternative method of atrial retraction compared with standard techniques of retraction. Minimal upgrades are needed to make it ready for human clinical trials. In addition to operator-dependent advantages such as ergonomics and ease of use, its surgical benefits compared with currently used devices include (1) equivalent exposure of the MVA; (2) subjectively more accessible left atrial volume, which could enable easier manipulation of surgical instruments within the chamber; (3) substantially less time to place; (4) minimal atriotomy incision length; and (5) customizable retraction.

Andy Richards, Christen Olafson, and Donna Hardin provided valuable assistance during porcine animal trials.

\section{References}

1. Kypson A. Recent trends in minimally invasive cardiac surgery. Cardiology. 2007; 107:147-58.

2. Cohn L, Adams D, Couper G, Bichell D, Rosborough D, Sears S, Aranki S. Minimally invasive cardiac valve surgery improves patient satisfaction while reducing costs of valve replacement and repair. Ann Surg. 1997;226:421-6.

3. Smith JM, Stein H, Engel A, McDonough S, Lonneman L. Totally endoscopic mitral valve repair using a robotic-controlled atrial retractor. Ann Thorac Surg. 2007; 84:633-7.

4. Akman A, Yilmaz G, Oto S, Akova Y. Comparison of various pupil dilation methods for phacoemulsification in eyes with a small pupil secondary to psuedoexfoliation. Opthalmology. 2004;111:1693-8.

5. Mishra YK, Malhotra R, Mehta Y, Sharma K, Kasliwal R, Trehan N. Minimally invasive mitral valve surgery through right anterolateral minithoracotomy. Ann Thorac Surg. 1999;68:1520-4.

6. Felger J, Chitwood WR, Nifong W, Holbert D. Evolution of mitral valve surgery: toward a totally endoscopic approach. Ann Thorac Surg. 2001;72:1203-9.

7. Jernigan S, Buckner G, Eischen J, Cormier D. Finite element modeling of the left atrium to facilitate the design of an endoscopic atrial retractor. $J$ Biomech Eng. 2007;129:825-37.

8. Gal O. fit_ellipse.m: Find the best fit for an ellipse using a given set of points (a closed contour) [Internet]. Natick (MA): The MathWorks, Inc, 2003 March 31 [cited 2007 July 31]. Available from: http://www.mathworks.com/matlabcentral/ fileexchange/loadFileList.do. 